

\title{
Permeability and pore connectivity: A new model based on network simulations
}

\author{
Y. Bernabé, M. Li, A. Maineult
}

\section{To cite this version:}

Y. Bernabé, M. Li, A. Maineult. Permeability and pore connectivity: A new model based on network simulations. Journal of Geophysical Research: Solid Earth, 2010, 115 (B10), pp.10203. 10.1029/2010JB007444 . insu-01729261

\section{HAL Id: insu-01729261 \\ https://hal-insu.archives-ouvertes.fr/insu-01729261}

Submitted on 12 Mar 2018

HAL is a multi-disciplinary open access archive for the deposit and dissemination of scientific research documents, whether they are published or not. The documents may come from teaching and research institutions in France or abroad, or from public or private research centers.
L'archive ouverte pluridisciplinaire HAL, est destinée au dépôt et à la diffusion de documents scientifiques de niveau recherche, publiés ou non, émanant des établissements d'enseignement et de recherche français ou étrangers, des laboratoires publics ou privés. 


\title{
Permeability and pore connectivity: A new model based on network simulations
}

\author{
Y. Bernabé, ${ }^{1} \mathrm{M} . \mathrm{Li}^{2}{ }^{2}$ and A. Maineult ${ }^{3}$ \\ Received 4 February 2010; revised 17 May 2010; accepted 8 June 2010; published 8 October 2010.
}

[1] The purpose of this paper is to model the effect of pore size heterogeneity and pore connectivity on permeability. Our approach is that of conceptual modeling based on network simulations. We simulated fluid flow through pipe networks with different coordination numbers and different pipe radius distributions. Following a method widely used in percolation theory, we sought "universal" relationships (i.e., independent of lattice type) between macroscopic properties such as permeability $k$ and porosity $\phi$, and, pore geometry attributes such as hydraulic radius $r_{\mathrm{H}}$, coordination number $z$, and so forth. Our main result was that in three-dimensional simple cubic, FCC, and BCC networks, permeability obeyed "universal" power laws, $k \propto\left(z-z_{\mathrm{c}}\right)^{\beta}$, where the exponent $\beta$ is a function of the standard deviation of the pore radius distribution and $z_{\mathrm{c}}=1.5$ is the percolation threshold expressed in terms of the coordination number. Most importantly, these power law relationships hold in a wide domain, from $z$ close to $z_{\mathrm{c}}$ to the maximum possible values of $z$. A permeability model was inferred on the basis of the power laws mentioned above. It was satisfactorily tested by comparison with published, experimental, and microstructural data on Fontainebleau sandstone.

Citation: Bernabé, Y., M. Li, and A. Maineult (2010), Permeability and pore connectivity: A new model based on network simulations, J. Geophys. Res., 115, B10203, doi:10.1029/2010JB007444.

\section{Introduction}

[2] Fluid flow through porous geologic materials plays an essential role in many geosciences fields such as hydrology or petroleum engineering. In practice, in situ information is often lacking, and there is a great need for models capable of predicting the permeability of rocks. However, permeability is very hard to model, owing to an extremely broad range of variation in the Earth (well over 11 orders of magnitude [Brace, 1980]) and because of the exceedingly heterogeneous nature of subsurface materials [e.g., Gelhar, 1993].

[3] Dimensional analysis shows that permeability $k$ is the product of a squared length scale $L^{2}$ and a dimensionless quantity $H$ :

$$
k=H L^{2} .
$$

The property expressed by equation (1) has been known for a very long time, but, to our knowledge, it was Berryman [1992a, 1992b, 1993] who most clearly stated that $H$ is not only dimensionless but also invariant under application to the porous medium of a uniform, isotropic scale transform (i.e., a transform such that the distance separating any pair of

\footnotetext{
${ }^{1}$ Earth, Atmospheric and Planetary Sciences Department, Massachusetts Institute of Technology, Cambridge, Massachusetts, USA.

${ }^{2}$ State Key Laboratory of Oil and Gas Reservoir Geology and Exploitation, Southwest Petroleum University, Chengdu, China.

${ }^{3}$ Institut de Physique du Globe de Paris, CNRS and Université Paris Cité, Paris, France.

Copyright 2010 by the American Geophysical Union. 0148-0227/10/2010JB007444
}

points in the medium is scaled by a constant factor). Porosity $\phi$ is the most common example of such a scale-invariant quantity (where "scale-invariant" is meant in the sense stated above and is not synonymous with "zoom-invariant," a different concept used in studies of fractal structures). Note that dimensionless quantities are not all scale-invariant. For example, the ratio of the electrical resistivity of the porous material to that of the saturating solution (often likened to the formation factor) is not scale-invariant unless the porous medium is uncharged and surface conduction is absent [Revil and Glover, 1997; Revil, 1999]. From the standpoint of equation (1), modeling permeability thus consists in answering the following questions. (1) For a given porous medium, what is the relevant length scale $L$ ? (2) Recognizing that $H$ must contain the contributions of many scaleinvariant parameters, which ones are the most important and in which functional form should they be included? This paper is an attempt to partially answer these two questions.

\section{Background}

[4] We first give a brief review of permeability models (see also Bernabé [1995] and Bernabé and Bruderer [1998]). The most common models are generically called Kozeny-Carman (KC) models. Their main characteristic is that they use the hydraulic radius $r_{\mathrm{H}}$ (i.e., twice the volumeto-surface ratio of the pores, $r_{\mathrm{H}}=2 V_{\mathrm{p}} / S_{\mathrm{p}}$, where $V_{\mathrm{p}}$ is the total pore volume and $S_{\mathrm{p}}$ the total wetted surface area). The principal reason for the popularity of $\mathrm{KC}$ models is that $r_{\mathrm{H}}$ is an easily measurable, purely geometric parameter (although smoothing of the wetted surface of the pores may sometimes 
be necessary; Blair et al. [1996]). One interesting KC model is the equivalent channel model (ECM) [Paterson, 1983; Walsh and Brace, 1984]:

$$
k=\frac{\phi}{b \tau^{2}} r_{\mathrm{H}}^{2},
$$

where $\tau$ is the tortuosity of the equivalent channel (i.e., the ratio of its actual to nominal length) and $b$ is a geometric factor equal to 8 if the pores are pipelike and 12 for thin cracks. Regarding $b$ as a simple constant, this model contains two scale-invariant parameters: porosity and tortuosity. The purely geometric definition of tortuosity mentioned above implies relatively low values of $\tau$ (say, from 1 to 3; for a detailed discussion of tortuosity, see Dullien [1979]). However, low tortuosities often lead to significant disagreement of equation (2) with rock experimental data. Because of this (among other things), a number of authors consider ECM unsatisfactory. It is often thought that, although $r_{\mathrm{H}}$ may be correctly measured and therefore geometrically representative of the porous medium considered, it is poorly relevant to the hydraulic flow problem. Two modifications of ECM are usually brought about: (1) the hydraulic radius is replaced by another, presumably more relevant length scale, and (2) the "electrical tortuosity" defined as the product $F \phi$, where $F$ is the electrical formation factor, is substituted for $\tau^{2}$. We do not try here to review all the attempts that have been made over the years, but focus on one important model by Johnson and co-workers [Johnson et al., 1987; Johnson, 1989; Johnson and Schwartz, 1989], who proposed the following length-scale:

$$
\Lambda=2 \frac{\int|E|^{2} \mathrm{~d} V_{\mathrm{p}}}{\int|E|^{2} \mathrm{~d} S_{\mathrm{p}}},
$$

where the local electrical field $E$ gives more weight to the strongly conducting pores than to the weakly conducting ones ( $E$ is the solution of an electrical conduction problem satisfying boundary conditions equivalent to the fluid flow ones). Using a weighted hydraulic radius such as $\Lambda$ does make sense once it is recognized that the pores in a heterogeneous porous medium are not all equally effective in conducting fluid flow (on the concept of effective and noneffective porosity, see also Bernabe et al. [2003b]). However, the drawback from the standpoint of equation (1) is that $\Lambda$ is not a purely geometric length-scale and cannot be measured directly (e.g., from microstructure observations). In fact, $\Lambda$ contains some of the scale-invariant parameters included in $H$ in equation (1).

[5] Our main goal in this paper is to follow another strategy. We wish to explicitly separate scale-variant and scale-invariant effects by defining $L$ in equation (1) as a strictly geometric length scale and by assigning all scaleinvariant parameters to $H$. As a consequence, question 1 loses some of its importance. Any geometrically representative length scale (e.g., mean pore length, mean pore radius, hydraulic radius, mean grain size, and so forth) is equally acceptable for our purpose. Here, we simply used the hydraulic radius $r_{\mathrm{H}}$. The fact that the choice of $L$ is arbitrary is not a problem. Let us consider two possible, purely geometric length scales $L_{1}$ and $L_{2}$. Clearly, their ratio $L_{1} / L_{2}$ is scale-invariant in the sense used here, and the associated $H_{1}$ and $H_{2}$ verify $H_{2}=H_{1}\left(L_{1} / L_{2}\right)^{2}$. Therefore, a change of length scale from $L_{1}$ to $L_{2}$ only entails the introduction into
$H$ of a $\left(L_{1} / L_{2}\right)^{2}$ factor. Thus, only question 2 remains. But it is an extremely hard question to answer because so many different scale-invariant effects are intricately entangled into $H$. We limit this study to trying to identify the most important ones and incorporate them separately and independently into $H$. In that regard, the "electrical tortuosity" mentioned above is as complex as $H$ itself [Dullien, 1979] and is therefore not appropriate for our purpose.

[6] Given the extreme complexity of fluid flow in porous media, we did not try here to assess all possible scaleinvariant effects to be included in $H$ but concentrated on only two that we think are of utmost importance: (1) pore size heterogeneity [e.g., Bernabé, 1995; Bernabé and Bruderer, 1998; Bernabé et al., 2003a] and (2) pore connectivity [e.g., Bernabé et al., 1982; Doyen, 1988; Zhang et al., 1994; Le Ravalec et al., 1996; Zhu et al., 1999; David et al., 1999; Fauzi et al., 2002]. These two effects can be unambiguously parameterized in rocks possessing a pore geometry such that a medial axis (i.e., reduction of the pore space to its underlying topological skeleton) can be measured [Thovert et al., 1994; Spanne et al., 1994; Lindquist et al., 1996; Baldwin et al., 1996; Fredrich and Lindquist, 1997; Lindquist and Venkatarangan, 1999; Fredrich, 1999; Lindquist et al., 2000; Petford et al., 2001; Sok et al., 2002]. This study is therefore limited to medial axis rocks. This limitation is grounded more in practice than in theory. Indeed, it should be possible, in principle, to define a medial axis for any porous medium (see, e.g., applications to biomaterials, Jones et al. [2009]). However, owing to limited resolution of the imaging technique used, the medial axis may be difficult to identify in rocks containing microporosity (i.e., submicron pores in sandstones and carbonates associated with, for example, clay or micritic calcite cement [Zinszner and Pellerin, 2007]). The medial axis method reduces the pore space to its underlying, topologically equivalent skeleton. In principle, this allows unambiguous identification of individual pores (defined by the segments joining nodes of the medial axis) and therefore quantitative determination of the statistical distributions of pore attributes such as length $l$, radius $r$, and so forth. Normalized standard deviations $\sigma_{l} /\langle l\rangle$, $\sigma_{r} /\langle r\rangle$, and so forth, of these geometric attributes are clearly scale-invariant parameters that can be included in $H$. Since the hydraulic conductance of an individual pore depends on the fourth power of $r$ and only on $l^{-1}$, we neglected the fluctuations in pore length in this paper and considered only $\sigma_{r} /\langle r\rangle$. We also neglected the variations in crosssectional surface area (i.e., changes of $r$ ) along individual pores. These assumptions imply that the variable denoted $r$ in $\sigma_{r} /\langle r\rangle$ refers to a local hydraulic radius. The medial axis method also permits measurement of the statistics of the coordination number (i.e., number of pores connected to a given node of the medial axis). Our contention is that the mean coordination number $z$ is a scale-invariant parameter measuring pore connectivity (fluid flow properties are not affected significantly by the fluctuations in local coordination number [Dullien, 1979; Sahimi, 1995]). In the past, pore connectivity has often been viewed from the standpoint of percolation theory. For example, Guéguen and Dienes [1989] defined connectivity as the ratio $f$ of the number of pores belonging to the percolating cluster to the total number of pores. This parameter is appropriate for describing the transport properties near the percolation 
Table 1. Expressions Relating $r_{\max }$ and $r_{\min }$ to $\sigma_{r} /\langle r\rangle$ and $r_{\mathrm{H}}$

\begin{tabular}{|c|c|}
\hline Log-Uniform & Uniform \\
\hline$r_{\mathrm{H}}=\frac{r_{\max }+r_{\min }}{2}$ & $r_{\mathrm{H}}=2 \frac{r_{\max }^{2}+r_{\max } r_{\min }+r_{\min }^{2}}{3\left(r_{\max }+r_{\min }\right)}$ \\
\hline$\frac{\sigma_{r}}{\langle r\rangle}=\sqrt{\frac{\log \left(r_{\max } / r_{\min }\right)\left(r_{\max }+r_{\min }\right)}{2\left(r_{\max }-r_{\min }\right)}-1}$ & $\frac{\sigma_{r}}{\langle r\rangle}=\frac{\left(r_{\max }-r_{\min }\right)}{\sqrt{3}\left(r_{\max }+r_{\min }\right)}$ \\
\hline
\end{tabular}

threshold $(f \approx 0)$ in tight rocks subjected to a random microcracking process (e.g., brittle straining, thermal shock, and so forth). But the important point here is that $f$ cannot differentiate between two fully connected porous media $(f \approx 1)$ with different mean coordination numbers but with equal geometrically representative length scales, although it can be easily verified (as was done here) that the medium with the highest coordination number is more permeable than the other one and therefore has a more connected pore space. Microstructure and experimental studies suggest that in upper crust rocks, disconnection of the pore space only occurs at low (sometimes very low) porosities [e.g., Madden, 1983; Mavko and Nur, 1997; Bernabé et al., 2003b]. Since we are primarily interested in the most common porous rocks (usually way above a possible percolation threshold), $z$ is more appropriate for our purpose than $f$. Furthermore, it should be noted that $z$ can also be used to quantify the approach to the percolation threshold. Earlier attempts to relate the transport properties of rocks to the coordination number include those of Dullien [1979], Doyen [1988], and, more recently, Fauzi et al. [2002].

\section{Numerical Procedures}

[7] Rocks possessing a medial axis can be conceptually represented as networks of pipes [Sok et al., 2002]. Network simulation is therefore a very convenient, conceptual modeling approach for studying the transport properties of these materials (for review, see Adler [1992]; Sahimi [1995]). Here, for the sake of simplicity, we only considered regular lattices of cylindrical pipes with circular crosssections. We simulated fluid flow through these networks by directly solving Kirchoff laws using periodic boundary conditions (for more details, see Bernabé and Bruderer [1998] and Bernabé et al. [2003a]). To widen the range of conceptual porous media explored, we considered twoand as well as three-dimensional lattices. In the case of two-dimensional networks, we must assign a thickness to the networks to calculate permeability and porosity. Here, we simply took it equal to $l$, the pipe length. According to this convention, perfectly homogeneous $\left(r=r_{\mathrm{H}}\right.$ for all pipes), two-dimensional square and three-dimensional simple cubic networks have an identical permeability, $k_{0}=$ $\pi r_{\mathrm{H}}^{4} /\left(8 l^{2}\right)$. With the values used here $\left(r_{\mathrm{H}}=40 \mu \mathrm{m}\right.$, a value typical of many high-porosity sandstones, and $l=300 \mu \mathrm{m}$, significantly greater than $r_{\mathrm{H}}$ to minimize the relative volume
Table 2. Values of $\sigma_{r} /\langle r\rangle, r_{\max }$ and $r_{\min }$ Used in This Study ${ }^{\mathrm{a}}$

\begin{tabular}{ccccccc}
\hline \multicolumn{3}{c}{ Log-Uniform } & & \multicolumn{3}{c}{ Uniform } \\
\cline { 1 - 2 } \cline { 5 - 6 }$\sigma_{r} /\langle r\rangle$ & $r_{\max }$ & $r_{\text {min }}$ & & $\sigma_{r} /\langle r\rangle$ & $r_{\text {max }}$ & $r_{\text {min }}$ \\
\hline 0.05 & 43.4563 & 36.5437 & & 0.05 & 43.3557 & 36.4448 \\
0.30 & 59.2411 & 20.7589 & & 0.30 & 55.7657 & 17.6288 \\
0.55 & 70.1570 & 9.84301 & & 0.55 & 59.9655 & 1.45480 \\
0.80 & 76.0414 & 3.95856 & & & \\
1.05 & 78.6472 & 1.35281 & & & \\
\hline
\end{tabular}

${ }^{\mathrm{a}}$ Radii are given in $\mu \mathrm{m}$. They all correspond to $r_{\mathrm{H}}=40 \mu \mathrm{m}$.

of pipe intersections) we obtain $k_{0}=11.210^{-12} \mathrm{~m}^{2}$. The network permeability values reported in the rest of this paper always correspond to the normalized permeability $k / k_{0}$.

[8] Pore-scale heterogeneity was generated by randomly choosing the pipe radii. Since microstructure studies suggest that the distributions of pore radii in rocks are strongly skewed [e.g., Fredrich and Lindquist, 1997; Lindquist et al., 2000], we used log-uniform distributions (i.e., uniform distribution of the $\log$ of $r$ ) with different values of the normalized standard deviation $\sigma_{r} /\langle r\rangle$ (namely, 0.05, 0.30, 0.55, 0.80 , and 1.05 ). For the sake of comprehensiveness, we also considered uniform distributions, although they are less relevant to rocks. Since $\sigma_{r} /\langle r\rangle$ cannot exceed 0.58 for uniform distributions, only the lowest three values of $\sigma_{r} /\langle r\rangle$ were used in this case. Each one of the $r$-distributions mentioned above is uniquely defined by the upper and lower limits, $r_{\max }$ and $r_{\min }$, of the domain of definition. For each value of $\sigma_{r} /\langle r\rangle$, we selected $r_{\max }$ and $r_{\min }$ in such a way that the hydraulic radius was constant, equal to $40 \mu \mathrm{m}$. Holding $r_{\mathrm{H}}$ constant ensures that the variations of permeability are only attributable to the scale-invariant $H$. The expressions relating $r_{\max }$ and $r_{\min }$ to $\sigma_{r} /\langle r\rangle$ and $r_{\mathrm{H}}$ are given in Table 1, and the values actually used in this study are listed in Table 2.

[9] Variations in coordination number were produced in two ways. (1) We used a variety of lattices, namely, twodimensional triangular, square, and hexagonal (also called honeycomb) and three-dimensional face-centered cubic (FCC), body-centered cubic (BCC), and simple cubic, with $z=6,4,2,12,8$ and 6 , respectively. (2) For each type of lattice, we also constructed realizations in which a number of bonds were randomly selected according to a probability $p$ and assigned a radius, whereas the remaining ones were left unfilled (i.e., $r=0$ ). It has been established previously that the transport properties of partially filled networks are not strongly affected by the variations in local coordination number but tend to be solely controlled by the mean coordination number $z$ [Dullien, 1979; Sahimi, 1995]. It is important to note that, in network simulations, $z$ is calculated over all the nodes of the network, even those with a local coordination number of zero. However, estimating the appropriate number of pores with a local coordination number of two or less may be very difficult in real porous media [Lindquist et al., 2000; Petford et al., 2001]. Figures $1 \mathrm{a}$ and $1 \mathrm{~b}$ show that, to a very good approximation, $z-z_{\mathrm{c}}$ was in all cases linearly related to $p-p_{\mathrm{c}}$,

Figure 1. Mean coordination number $z$ as a function of probability of occupancy $p$. The black straight lines join the points $\left\{p=1, z_{\max }\right\}$ on the right side of the diagram to $\left\{p_{\mathrm{c}}, z_{\mathrm{c}}\right\}$ (marked by arrows and the gray horizontal line). (a) 2-D lattices (Tri, Sq, and Hex refer to triangular, square, and hexagonal, respectively). (b) 3-D lattices (FCC, BCC, and SC refer to facecentered cubic, body-centered cubic, and simple cubic, respectively). 


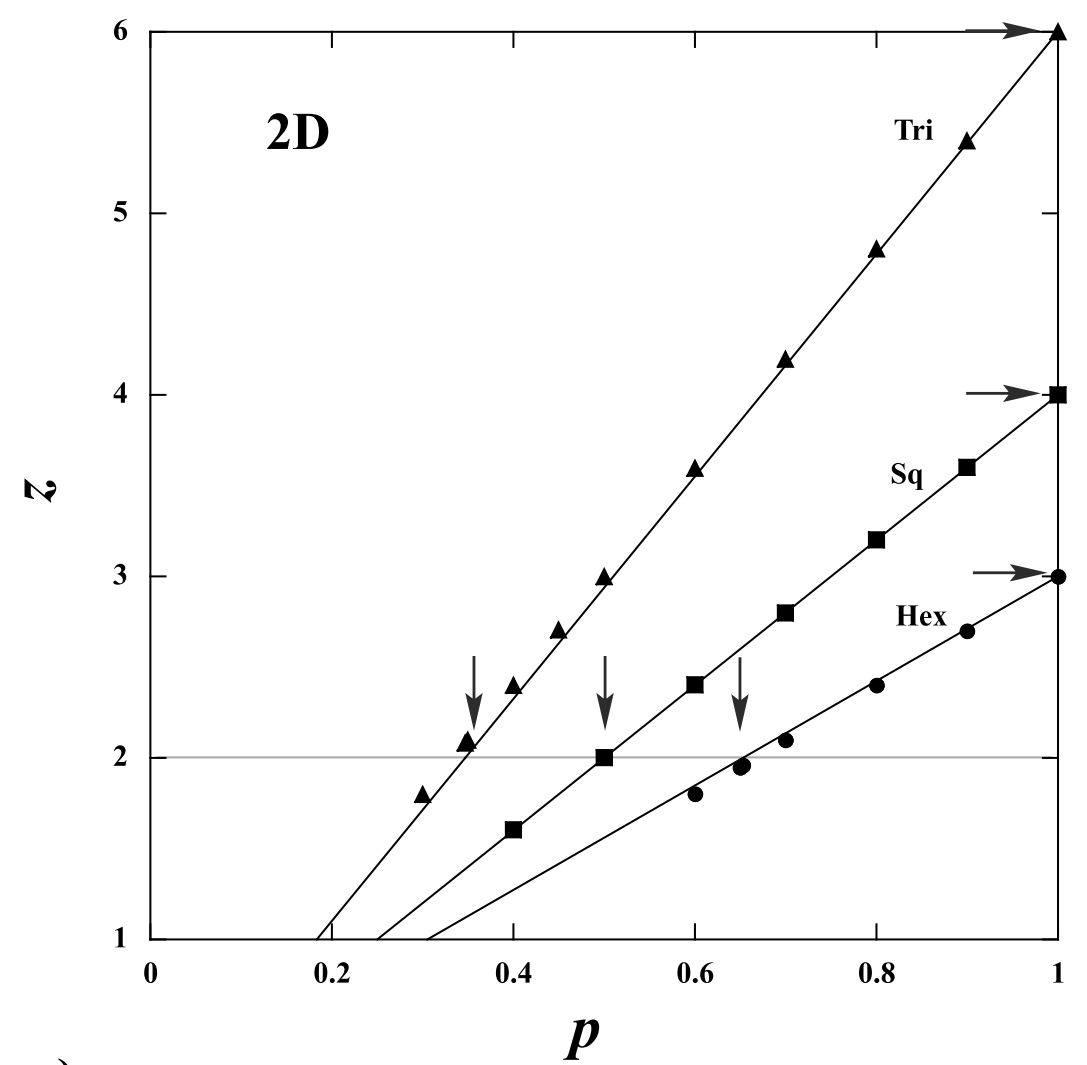

a)

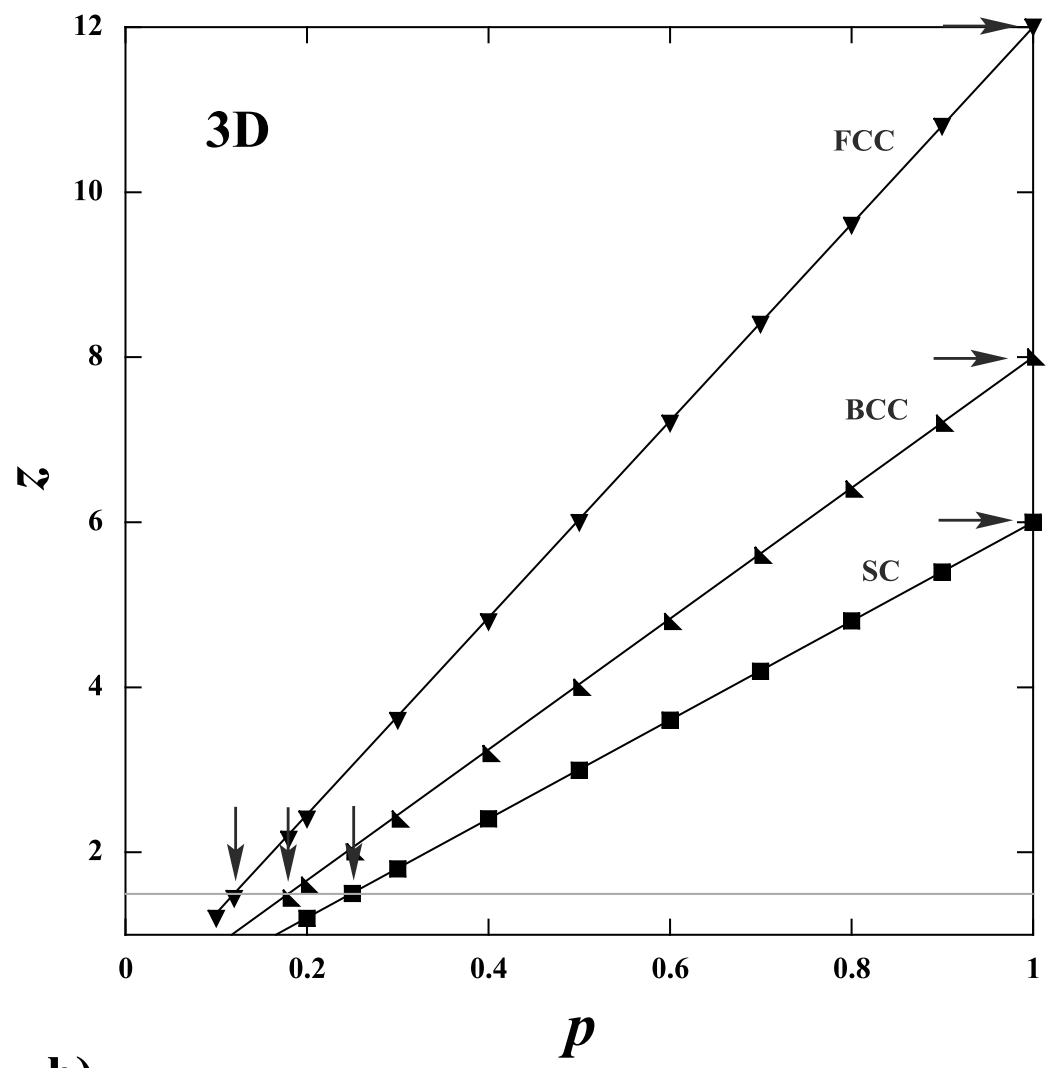

b)

Figure 1 
where $p$ is the probability of occupancy of a pipe, $p_{\mathrm{c}}$ the bond percolation threshold, and $z_{\mathrm{c}}$ the critical coordination number (i.e., the percolation threshold in terms of coordination number). As a visual proof of this statement, we plotted the straight lines joining the theoretical end points, $\left\{p=1, z_{\max }\right\}$ and $\left\{p_{\mathrm{c}}, z_{\mathrm{c}}\right\}$, marked by arrows in Figures 1a and $1 \mathrm{~b}$. These lines fit the numerical results very well, although they were not calculated using a best-fit algorithm. In our network simulations, the independent variable $p-p_{\mathrm{c}}$ is not "universal" in the sense of percolation theory ( $p_{\mathrm{c}}$ depends on lattice type). The linear relationship mentioned above allows us to replace it with $z-z_{\mathrm{c}}$, a "universal" quantity permitting comparison of the different lattices. The values of $p_{\mathrm{c}}$ are known to be $0.5,0.347$, $0.653,0.249,0.180$, and 0.120 for the square, triangular, hexagonal, simple cubic, BCC, and FCC networks, respectively, while $z_{\mathrm{c}} \approx 2$ and 1.5 in two- and threedimensional lattices, respectively [e.g., Sahimi, 1995].

[10] Porosity $\phi$ is obviously a linear function of $p$ and therefore must also be linearly related to $z$. However this relationship is not universal in the sense of percolation theory; that is to say, it varies with lattice type. We did verify this property (as well as the nonuniversality of the relationship of $\phi$ with $k$ ), but, to save space, we do not illustrate it here with specific figures. The implication is that we must consider porosity to be a dependent function of $z-z_{\mathrm{c}}$ and $\sigma_{r} /\langle r\rangle$, just like permeability.

[11] Determining the permeability of partially filled networks poses a technical problem: When a sufficiently large number of bonds are unfilled, the network matrix becomes singular. Finding the appropriate lower rank matrix can be done, but it is easier to assign a very small, finite value $r_{0}$ to the radius of the unfilled bonds [Kirkpatrick, 1971] such that the corresponding permeability accurately approximates that of the actual partially filled network. In general, we found that a satisfactory value was $r_{0}=r_{\min } / 100$ (we started with $r_{0}=r_{\min } / 10$ and tried lower and lower values until a constant limit of $k$ was reached within an accuracy of $10^{-6}$ ). For $z$ very near $z_{\mathrm{c}}, r_{\min } / 100$ was seldom sufficiently small and further reductions of $r_{0}$ were implemented, often causing the network matrix to become numerically singular. In these last cases, we had to return to the original value $r_{0}=r_{\min } / 100$, although a numerical accuracy of $10^{-6}$ could not be maintained.

[12] It is well known that statistical fluctuations in permeability decrease with the size of the network realizations. Using the total number of bonds $n_{\mathrm{p}}$ as a measure of network size, Bernabé et al. [2003a] found statistical fluctuations in $k<1 \%$ for networks of size $>4000$, when they were moderately heterogeneous $\left(\sigma_{r} /\langle r\rangle=0.5\right.$ or lower). For $\sigma_{r} /\langle r\rangle=1.05$, the $k$ fluctuations reached values as large as $10 \%$ [Bernabé et al., 2003a]. In the present work, we used $60 \times 60$ two-dimensional networks, giving $n_{\mathrm{p}}=10,800,7200$, and 5400 for triangular, square, and hexagonal networks, respectively. The three-dimensional networks were $15 \times 15 \times 15$ for the simple cubic $\left(n_{\mathrm{p}}=\right.$ $10,125), 12 \times 12 \times 12$ for FCC $\left(n_{\mathrm{p}}=41,472\right)$, and $14 \times$ $14 \times 14$ for BCC $\left(n_{\mathrm{p}}=21,952\right)$. We observed $k$ fluctuations in general agreement with the results of Bernabe et al. [2003a], except that partially filled networks tended to have greater fluctuations. Even though we are not primarily interested by the approach to the percolation threshold, we also conducted calculations for $z$ very close to $z_{\mathrm{c}}$, but large fluctuations occurred as expected from percolation theory. Thus, the ensemble-averaged results in these cases had a greater uncertainty than for $z>1.2 z_{\mathrm{c}}$ or so (e.g., we observed relative $k$ fluctuations as high as $20 \%$ for $\sigma_{r} /\langle r\rangle=$ 1.05 and $z<1.2 z_{\mathrm{c}}$ ). The results reported in the next section are ensemble mean values $\langle k\rangle$ for 200 independent realizations for each case considered. Given the $k$ fluctuations mentioned above, the relative uncertainty on $\langle k\rangle$ was on the order of $1 \%$ or lower in all cases, except when $z$ was $<1.2 z_{\mathrm{c}}$.

[13] We also calculated the porosity $\phi$ of each network realization as the ratio of the total volume of the pipes (counting zero for unfilled bonds) to the total volume of the networks (in two-dimensional networks, we used the same convention as for $k$ and defined the network volume as the product of the total surface area of the network and the pipe length $l$ ). In all cases, we found the relative $\phi$-fluctuations to be small compared with the corresponding $k$ fluctuations (i.e., on the order of $2 \%$ or lower).

\section{Results}

[14] Using the numerical techniques explained above, we obtained ensemble mean values of permeability and porosity (hereafter, simply denoted $k$ and $\phi$ ) for two- and threedimensional networks with $\sigma_{r} /\langle r\rangle$ varying from 0.05 to 1.05 and $z$ covering the entire range from $z_{\max }$ the nominal values for each type of lattice to nearly $z_{\mathrm{c}}$. We found that normalized permeability $k / k_{0}$ decreased with decreasing $z-z_{\mathrm{c}}$ and with increasing $\sigma_{r} /\langle r\rangle$. As is commonly done in percolation theory, we drew $\log$-log plots of $k / k_{0}$ versus $z-z_{\mathrm{c}}$ (at constant $\left.\sigma_{r} /\langle r\rangle\right)$. The linearity of these plots was excellent in all cases (especially if only the values corresponding to $z-z_{\mathrm{c}}>0.4$ were used) demonstrating power law relationships between $k / k_{0}$ and $z-z_{\mathrm{c}}$. Figure 2 shows examples for twodimensional networks with $\sigma_{r} /\langle r\rangle=0.3$, and, as we can see, these power laws $k / k_{0} \propto\left(z-z_{\mathrm{c}}\right)^{\beta}$ display a significant dependence on lattice type. But this dependence essentially disappears if the product $\left(k / k_{0}\right) \phi$ is plotted instead of $k / k_{0}$. In other words, for each value of $\sigma_{r} /\langle r\rangle$, the $\left(k / k_{0}\right) \phi$ results obtained for the triangular, square, and hexagonal lattices can be superposed to define a single universal power law. For the sake of completeness, we examined other combinations of $k$ and $\phi$, namely $k / \phi, k, k \phi, k \phi^{2}$, and $k \phi^{3}$, and estimated the misfit with power law models as the RMS of the difference $\log \left(k_{\mathrm{obs}}\right)-\log \left(k_{\mathrm{mod}}\right)$, where the subscripts "obs" and "mod," respectively, refer to the values observed in the network simulations and to the power law models. We obtained $0.299,0.188,0.128,0.193$, and 0.310 for $k / \phi$, $k, k \phi, k \phi^{2}$, and $k \phi^{3}$, in that order. Thus, in two dimensions, the product $k \phi$ appears to be the combination of $k$ and $\phi$ most independent on lattice type, suggesting that the corresponding power law, $\left(k / k_{0}\right) \phi \propto\left(z-z_{\mathrm{c}}\right)^{\gamma}$, where the exponent $\gamma$ is a function of $\sigma_{r} /\langle r\rangle$, is the most likely to be applicable to general two-dimensional porous media (e.g., partially closed rough fractures). We conducted a similar analysis of the three-dimensional results and found misfits of $0.364,0.215,0.117,0.191$, and 0.333 for $k / \phi^{2}, k / \phi, k, k \phi$, and $k \phi^{2}$, respectively. As suggested by the minimum misfit occurring for $k$, the power law, $k / k_{0} \propto\left(z-z_{\mathrm{c}}\right)^{\beta}$, where the exponent $\beta$ is a function of $\sigma_{r} /\langle r\rangle$, is essentially independent on three-dimensional lattice type and therefore can reason- 


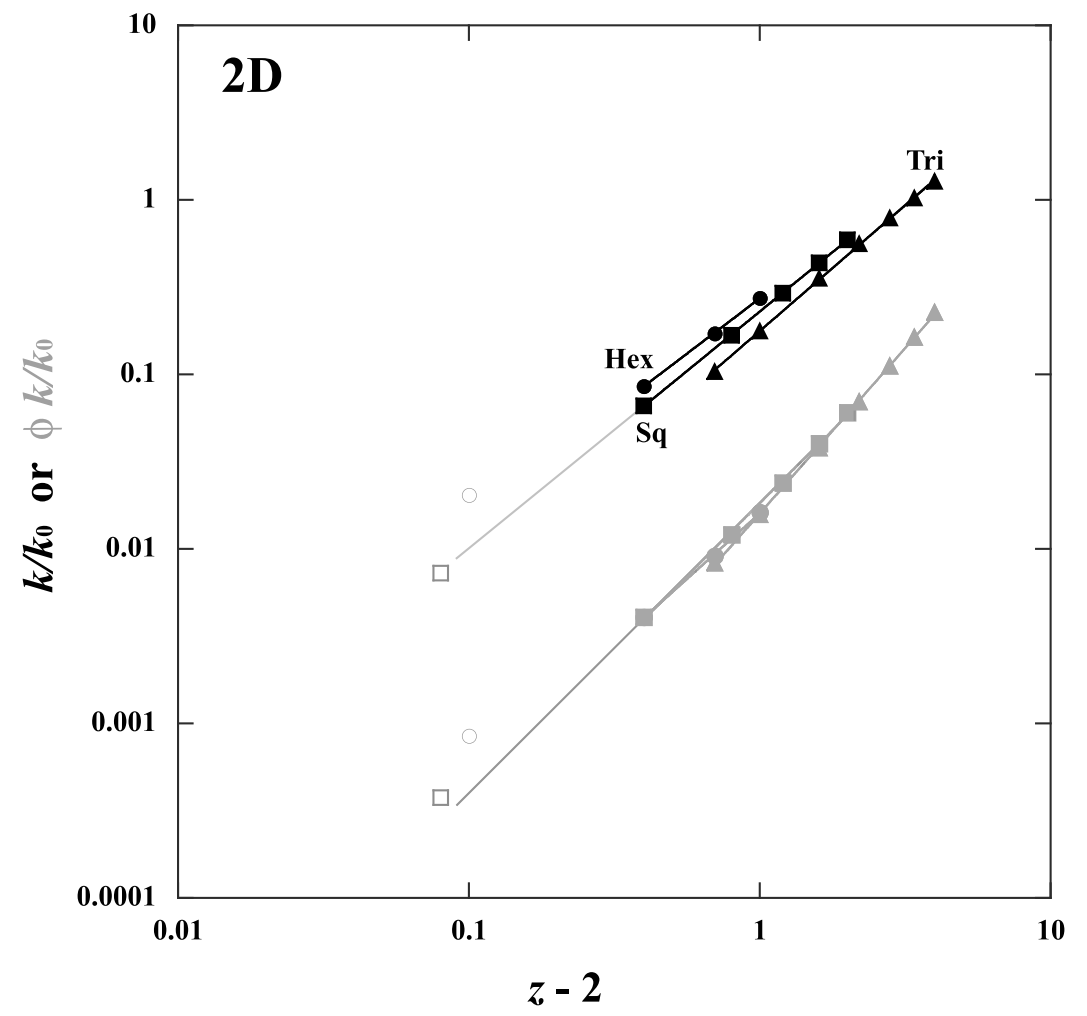

Figure 2. An example of log-log plots of $k$ (black) and $k \phi$ (gray) versus $z-2$ for the 2-D networks (with $\sigma_{r} /\langle r\rangle=0.30$ ). The straight lines indicate the individual best-fit power laws for each lattice (triangular, square, and hexagonal lattices indicated by triangular, square, and circular symbols, respectively, as well as by the labels Tri, Sq, and Hex). The power laws for $k$ are clearly lattice-specific, whereas those for $k \phi$ can be superposed independently on lattice type. The open symbols denote inaccurate data points close to the percolation threshold (see section 4).

ably be expected to apply to general three-dimensional porous media. The fact that different results are obtained in two- and three-dimensional networks is a very common phenomenon in percolation theory studies [e.g., Adler, 1992; Sahimi, 1995].

[15] The power laws $\left(k / k_{0}\right) \phi \propto\left(z-z_{\mathrm{c}}\right)^{\gamma}$ and $k / k_{0} \propto\left(z-z_{\mathrm{c}}\right)^{\beta}$ for two- and three-dimensional networks, respectively, are shown in Figures 3 and 4, where only the simulations corresponding to the log-uniform $r$ distributions are plotted. Similar well-fitting power laws were also observed for the uniform distributions. Note that the statistically uncertain data points corresponding to $z-z_{\mathrm{c}}<0.4$ (represented by open symbols in Figures 3 and 4) were not included in the determination of the power laws. The measured values of the exponents $\gamma$ and $\beta$ are plotted versus $\sigma_{r} /\langle r\rangle$ in Figures 5 and 6. In two-dimensional networks, the exponent $\gamma$ increased from 1.5 to 3.9 for log-uniform $r$-distributions with $\sigma_{r} /\langle r\rangle$ increasing from 0.05 (near homogeneity) to 1.05 (very strong heterogeneity). The uniform $r$ distributions did not yield the same values of $\gamma$ as the log-uniform ones. As $\sigma_{r} /\langle r\rangle$ was increased from 0.05 to 0.55 , the discrepancy grew from zero to about $1 / 2$. In three-dimensional networks, the exponent $\beta$ increased from 1.3 to 3.8 as $\sigma_{r} /\langle r\rangle$ varied in the same range as before. Contrary to the two-dimensional case, $\log$-uniform and uniform $r$ distributions produced numerically identical values of $\beta$, making it even more likely that the power laws $k / k_{0} \propto\left(z-z_{\mathrm{c}}\right)^{\beta}$ shown in Figure 4 are applicable to general three-dimensional porous media. Finally, we emphasize that the power laws reported above should not be confused with the critical power law of $k$ with respect to $p-p_{\mathrm{c}}$, which, percolation theory shows, is universal (i.e., independent of lattice type [Kirkpatrick, 1971; Sahimi, 1995]) in a close vicinity of $p_{\mathrm{c}}$. Our results demonstrate another kind of universality, namely, the (very near) independence on lattice type of the power laws of $\phi k$ or $k$ with $z-z_{\mathrm{c}}$ in the entire range of $z$ (except possibly very close to $z_{\mathrm{c}}$ ). This later type of universality, which to our knowledge escaped the attention of previous researchers, holds for a greater range of materials and is therefore of greater relevance to geology and geophysics problems than the former one. It appears to be linked to the fact that the percolation threshold expressed in terms of the coordination number is very nearly universal (i.e., $z_{\mathrm{c}} \approx 2$ and 1.5 in all two- and three-dimensional lattices, respectively). This observation shows the importance of $z$ as a parameter for characterizing pore connectivity in rocks.

\section{Inferred Model and Comparison With Experimental Data}

[16] We know that networks of pipes are not realistic representations of porous rocks even if they are constructed with realistic distributions of pore radii. However, as conceptual models of porous media, they can provide helpful 




Figure 3. Superposed $\log -\log$ plots of $k \phi$ versus $z-2$ for all 2-D lattices. Each type of symbol represents a different value of $\sigma_{r} /\langle r\rangle$ as indicated on the diagram. The straight lines show the best-fit power law in each case. The open symbols denote inaccurate data points close to the percolation threshold, which were not included in the power law fitting calculations.

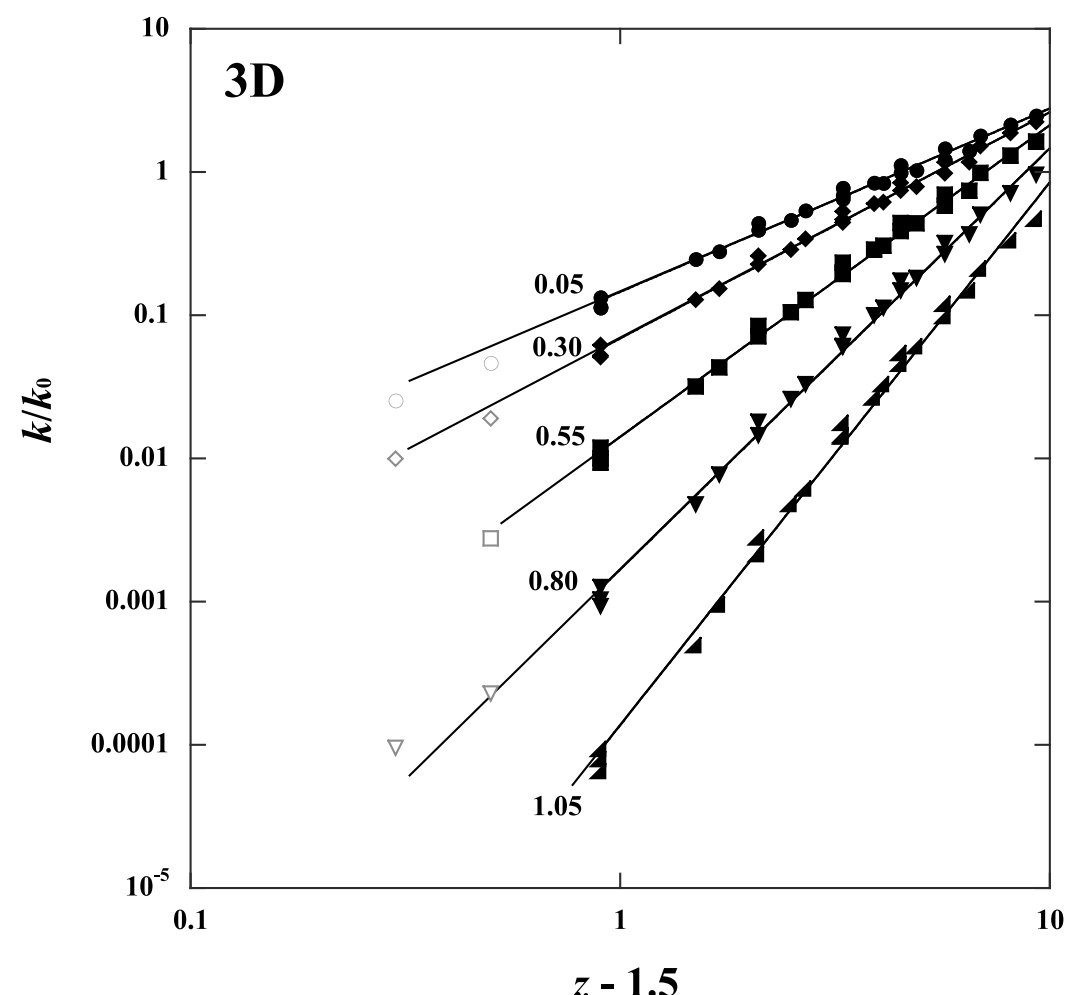

Figure 4. Superposed log-log plots of $k$ versus $z-1.5$ for all 3-D lattices. Each type of symbol represents a different value of $\sigma_{r} /\langle r\rangle$ as indicated on the diagram. The straight lines show the best-fit power law in each case. The open symbols denote inaccurate data points close to the percolation threshold, which were not included in the power law fitting calculations. 


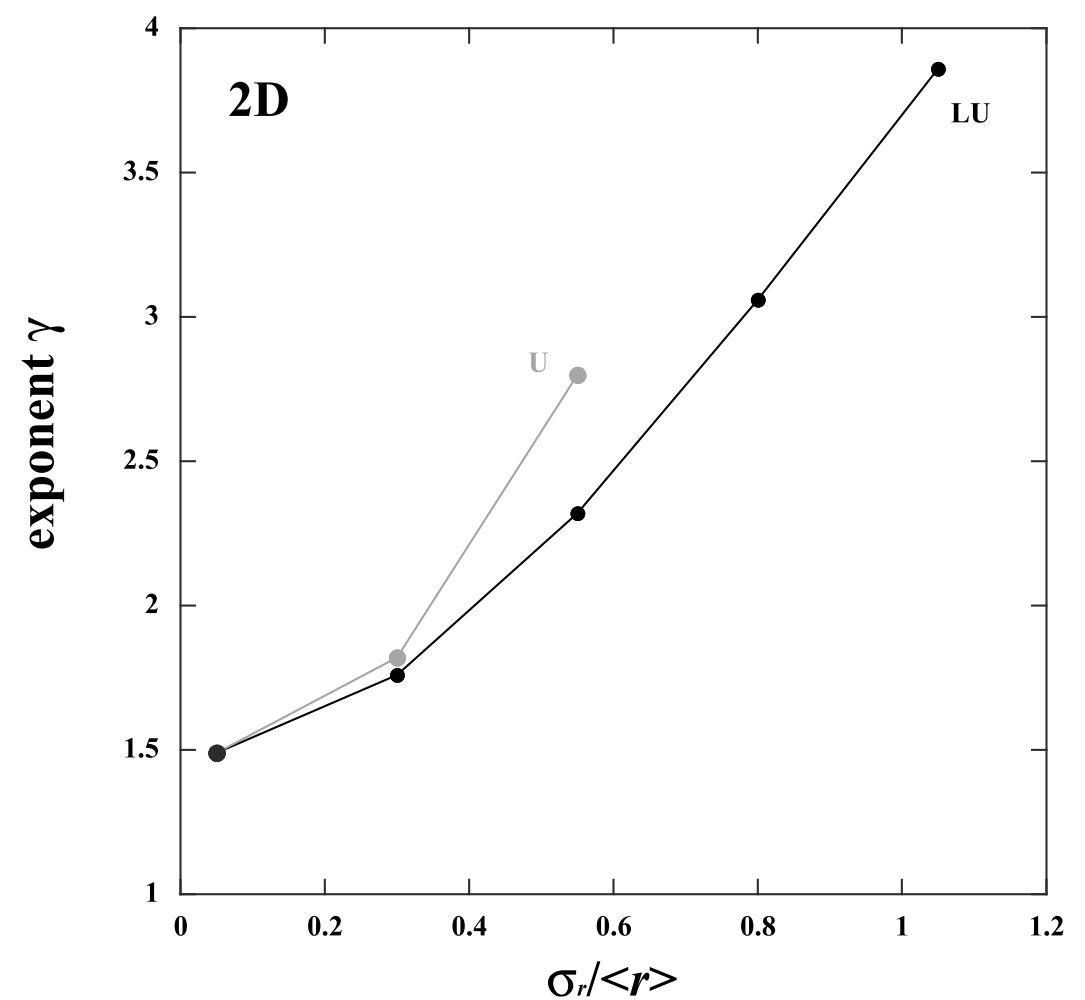

Figure 5. The exponent $\gamma$ of the power laws $\left(k / k_{0}\right) \phi \propto(z-2)^{\gamma}$ as a function of $\sigma_{r} /\langle r\rangle$. These power laws were calculated in 2-D networks with uniform (gray label U) and log-uniform (black label LU) distributions of pipe radii. Note that the $\mathrm{U}$ and LU values do not coincide at high $\sigma_{r} /\langle r\rangle$.

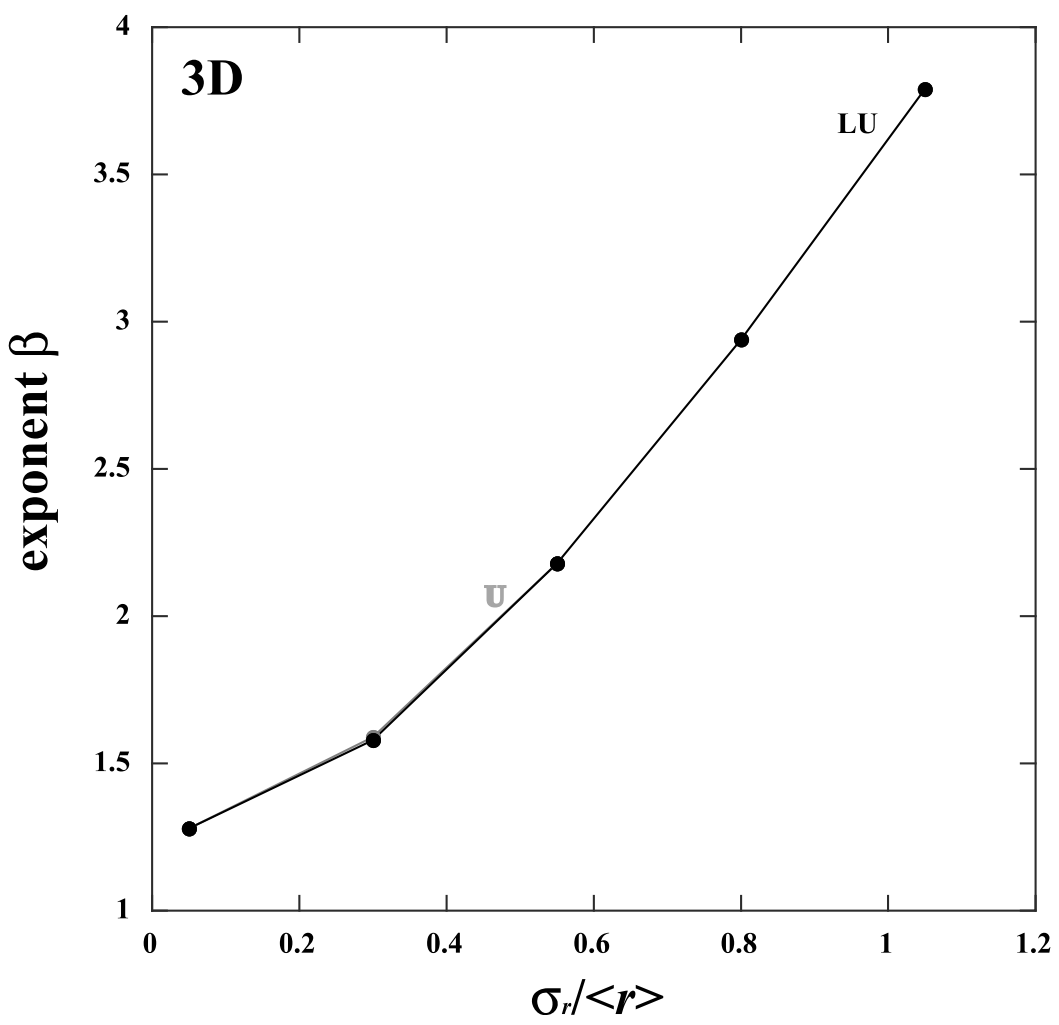

Figure 6. The exponent $\beta$ of the power laws $k / k_{0} \propto(z-1.5)^{\beta}$ as a function of $\sigma_{r} /\langle r\rangle$. These power laws were calculated in 3-D networks with uniform (gray label U) and log-uniform (black label LU) distributions of pipe radii. Note that the $\mathrm{U}$ and LU values coincide for all values of $\sigma_{r} /\langle r\rangle$. 


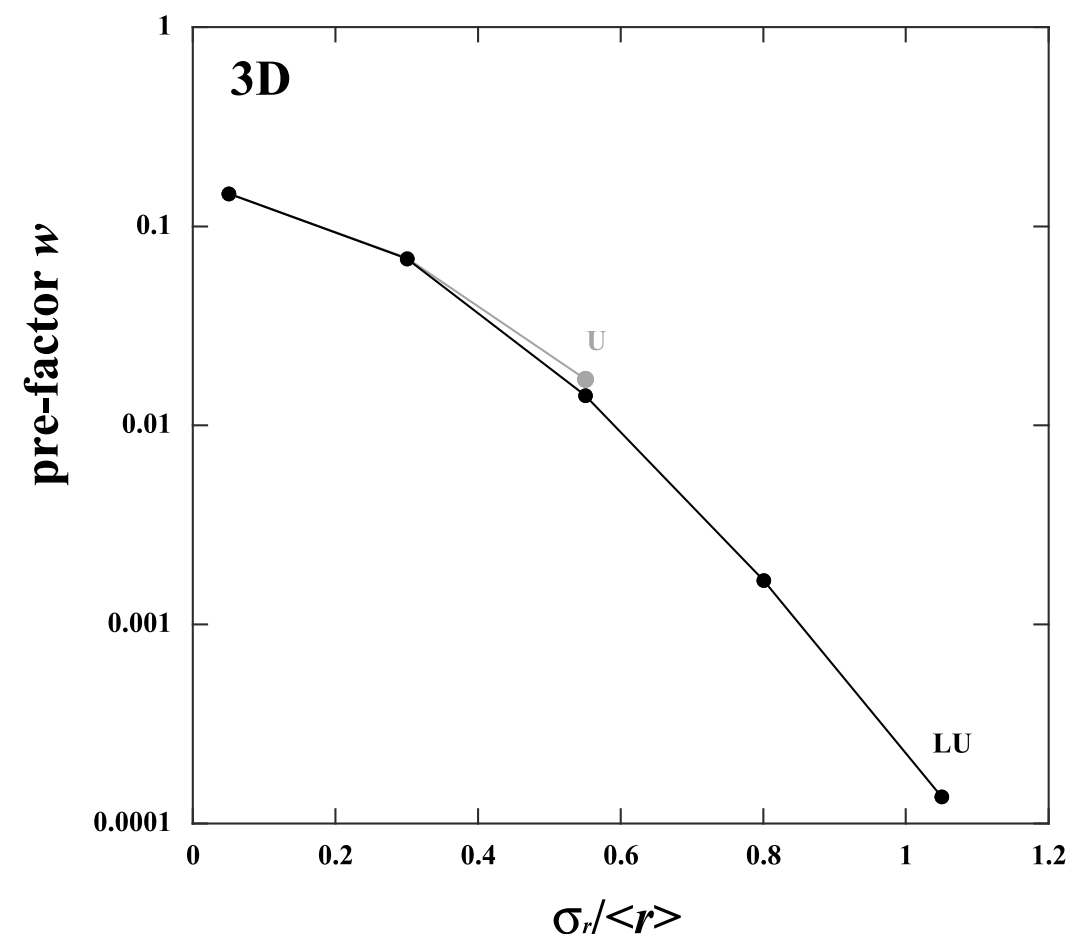

Figure 7. The pre-factor $w$ of the power laws $k / k_{0} \propto(z-1.5)^{\beta}$ as a function of $\sigma_{r} /\langle r\rangle$. These power laws were calculated in 3-D networks with uniform (gray label U) and log-uniform (black label LU) distributions of pipe radii. Note that the $\mathrm{U}$ and $\mathrm{LU}$ values nearly coincide for all values of $\sigma_{r} /\langle r\rangle$.

insight for identifying relations among statistical microstructure attributes of porous media (considered as independent variables) and macroscopic properties such as permeability and porosity (considered as dependent functions). The problem with this method is to identify the network properties that can reasonably be generalized to real porous media. One approach (followed here) is to reject the lattice-specific features and retain the "universal" results (i.e., the ones which do not depend on lattice type). Our results suggest that (three-dimensional) porous media possessing a medial axis should follow the power law, $k=$ $w k_{0}\left(z-z_{\mathrm{c}}\right)^{\beta}$, where the prefactor $w$ and exponent $\beta$ are functions of $\sigma_{r} /\langle r\rangle$. Given the definition of $k_{0}$, we end up with the following model:

$$
k=w \frac{\pi}{8}\left(\frac{r_{\mathrm{H}}}{l}\right)^{2}(z-1.5)^{\beta} r_{\mathrm{H}}^{2} .
$$

The observed variations of $\beta$ and $w$ with $\sigma_{r} /\langle r\rangle$ displayed in Figures 6 and 7 do not obey a simple functional form and must be incorporated numerically into equation (4). There are three scale-invariant parameters in this model: (1) the mean coordination number $z$, which measures pore connectivity; (2) through its control of $\beta$ and $w$, the normalized standard deviation of the pore radius distribution $\sigma_{r} /\langle r\rangle$, which measures pore size heterogeneity; and (3) the ratio $r_{\mathrm{H}} / l$, which can be interpreted as the ratio of mean pore radius to mean pore separation distance (alternatively, it could be viewed as a sort of longitudinal pore aspect ratio). Even though the proposed model includes $r_{\mathrm{H}}$ as length scale, it is quite different from $\mathrm{KC}$ models, which treat porosity as an independent variable like $r_{\mathrm{H}}$ or $z$ (e.g., see equation (2)), whereas in this study, $\phi$ is a dependent parameter like $k$.
[17] Experimental data sets against which to test our model are rare. In fact, we had to merge the Fontainebleau sandstone data of Doyen [1988], Fredrich et al. [1993] and Lindquist et al. [2000]. All three papers present experimental and microstructure data on Fontainebleau sandstone samples covering the same range of porosity (roughly from $4 \%$ to $22 \%$ ). Doyen [1988] measured $\phi, k$, and the electrical formation factor $F$ of rock cores and conducted microstructure analysis on (two-dimensional) thin sections. He determined histograms of the throat and nodal pore radii and estimated the coordination number $z_{2 \mathrm{D}}$ using a rather contrived extrapolation technique (in principle, $z$ is not accessible in two-dimensional images). Fredrich et al. [1993] also measured $\phi, k$, and $F$. They determined the hydraulic radius $r_{\mathrm{H}}$ from quantitative strereology analysis of stacked twodimensional images obtained by laser confocal microscopy. Permeability data from Doyen [1988] and Fredrich et al. [1993] are plotted versus porosity in Figure 8. We can see clearly that these two data sets correspond to a single rock type and can therefore be meaningfully merged together. Lindquist et al. [2000] measured the porosity of rock samples and carried out the medial axis analysis of three-dimensional $\mathrm{X}$-ray microtomographic images obtained from them. They determined the distributions of pore lengths, throat crosssectional areas and nodal pore volumes. They also measured the distribution of local coordination numbers but, for practical reasons, had to use a different definition. Indeed, in the form implemented by Lindquist et al. [2000], the medial axis method did not allow unambiguous identification of nodal pores with a local coordination number of 2 . As a consequence, Lindquist et al. [2000] decided to exclude from their analysis the nodes with local coordination numbers 


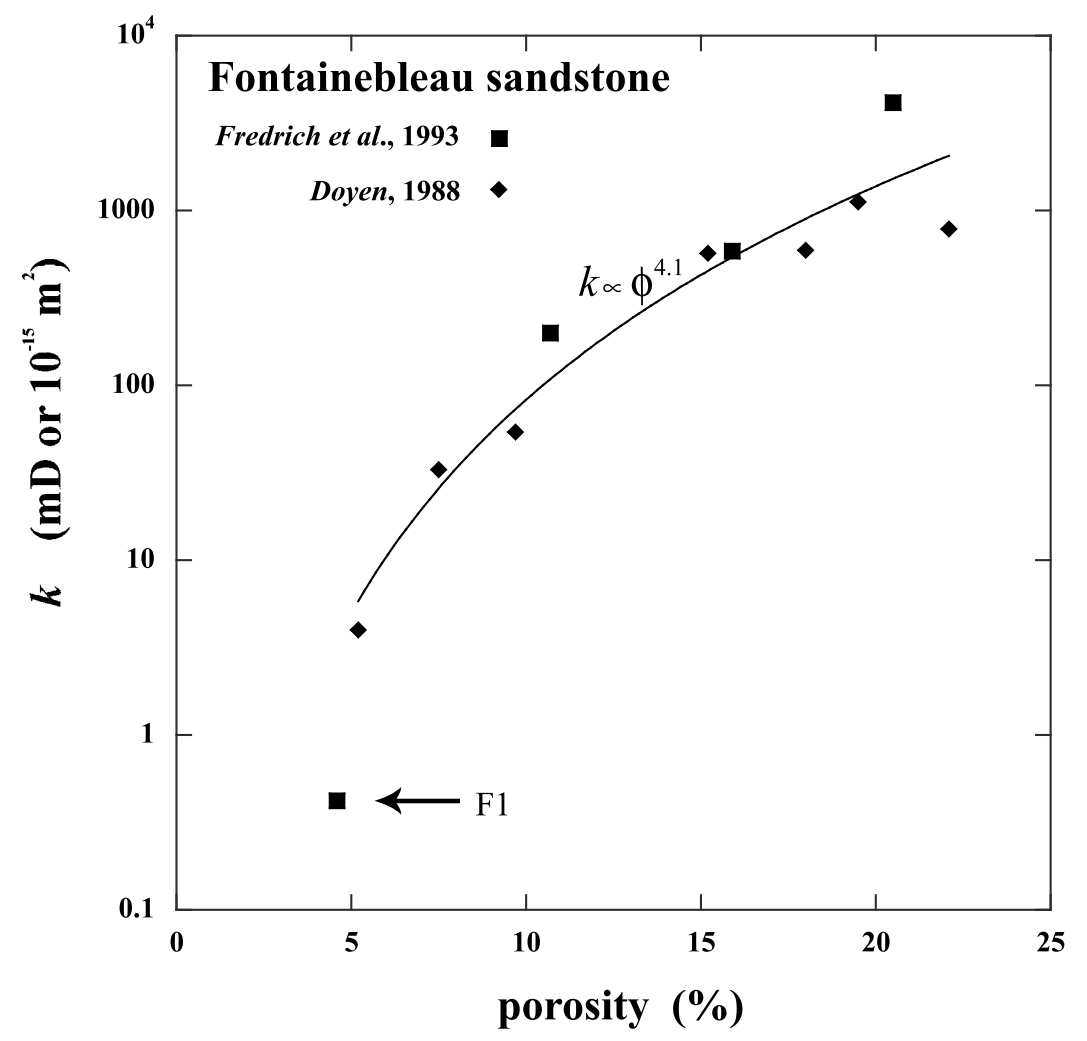

Figure 8. Permeabilities measured in Fontainebleau sandstone samples by Doyen [1988] (diamonds) and Fredrich et al. [1993] (squares) plotted versus porosity. The curved line corresponds to the best-fit power law $k \propto \phi^{4.1}$. This power law was established using the two data sets with the exception of sample F1 (indicated by arrow), because it is too close to the percolation threshold.

lower than or equal to 2 . This practice yields a modified mean coordination number $z^{*}$, a parameter always greater than 3 , that has not been intensively studied before. In particular, the critical value $z^{*}{ }_{\mathrm{c}}$ corresponding to the percolation threshold is unknown.

[18] To test equation (4), we tried to complete the data sets of Doyen [1988] and Fredrich et al. [1993] using porosity transforms for estimating the missing parameters. From Lindquist et al.'s [2000] $l, z^{*}$, and $\phi$ data, we constructed $l$ versus $\phi$ and $z^{*}$ versus $\phi$ formulas, whereas we used Fredrich et al. [1993] for $r_{\mathrm{H}}$ versus $\phi$ and Doyen [1988] for $z_{2 \mathrm{D}}$ versus $\phi$. These formulas (listed in Table 3) were then used to transform the $\phi$ data of Doyen [1988] and Fredrich et al. [1993] into the missing $l, z^{*}, r_{\mathrm{H}}$, and $z_{2 \mathrm{D}}$ data sets. The transforms of Table 3 were selected for goodness of fit as well as simplicity of mathematical formula. They should not, by any means, be considered the only ones possible. In addition, we simultaneously determined $z^{*}$ and $z$ in the network realizations used for flow simulation. The results are shown in Figure 9. For $z>6$, we verified that $z^{*} \approx z$ (indeed, nodes with coordination numbers of 2 or less are very rare in highly connected networks with $z>6$ ), whereas for $z<6$, the difference $z^{*-} z$ was found to vary approximately as $\log _{10}(z)$. We selected two nearly identical transforms (see Figure 9 and Table 3 ) that essentially differed by the critical value $z^{*}{ }_{\mathrm{c}}$ reached for $z=z_{\mathrm{c}}$ (we found 3.21 and 3.23 for transforms 1 and 2, respectively). Again, there may be other transforms just as accurate and/or convenient as the ones shown in Figure 9.

[19] Next, we needed to estimate $\sigma_{r} /\langle r\rangle$ for Fontainebleau sandstone and infer the corresponding values of exponent $\beta$ and prefactor $w$. From the histograms published by Doyen [1988], we approximately calculated the normalized standard deviations for throat size $\left(\sigma_{\text {throat }}\right)$ and nodal pore radius $\left(\sigma_{\text {nodal }}\right)$. We found $\sigma_{\text {throat }} \approx 0.7$ and 0.8 and, $\sigma_{\text {nodal }} \approx 0.4$ and 0.6 for the samples with $7.5 \%$ and $22.1 \%$ porosity, respectively. We speculate that these values are likely to overestimate $\sigma_{r} /\langle r\rangle$ because they are affected not only by the

Table 3. Transforms Used to Complete the Data Sets of Doyen [1988] and Fredrich et al. [1993]

\begin{tabular}{ll}
\hline \multicolumn{1}{c}{ Transform } & Comment \\
\hline$\langle l\rangle=457.14 \phi^{-0.39752}$ & $(\phi$ in \%; based on Lindquist et al. [2000]) \\
$z^{*}=3.1131+0.029929 \phi$ & $(\phi$ in \%; based on Lindquist et al. [2000]) \\
$r_{\mathrm{H}}=13.2-0.24651 \phi+0.027136 \phi^{2}$ & $(\phi$ in \%; based on Fredrich et al. [1993]) \\
$z_{2 \mathrm{D}}=-2.1249+6.2455 \log ^{10} \phi$ & $(\phi$ in \%; based on Doyen [1988]) \\
$z^{*}-z=2.1867-2.7078 \log _{10} z$ & (transform \#1; $z^{*}=3.21 ;$ from Figure 8) \\
$z^{*}-z=2.2373-2.8627 \log _{10} z$ & (transform \#2; $z_{\mathrm{c}}^{*}=3.23 ;$ from Figure 8) \\
\hline
\end{tabular}




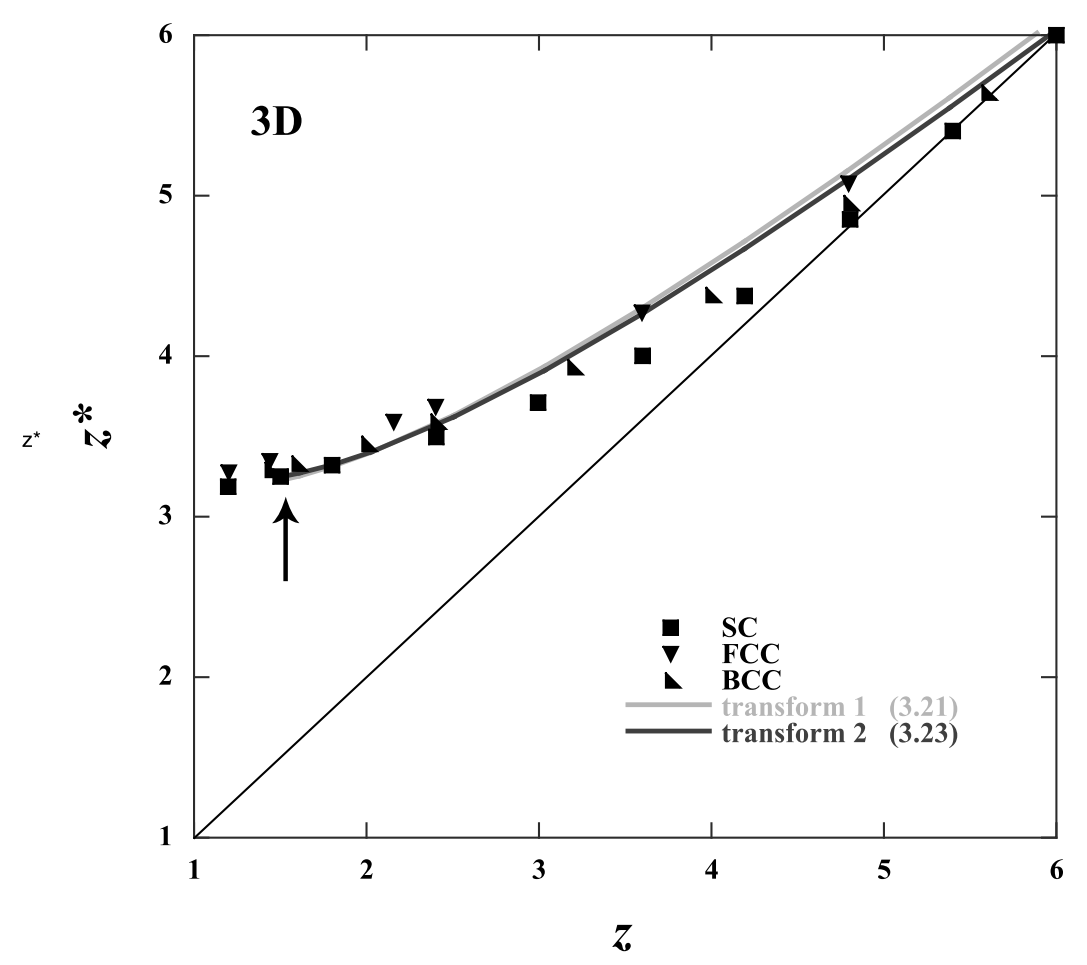

Figure 9. The modified mean coordination number $z^{*}$ plotted versus the mean coordination number $z$ (both simultaneously measured in the 3-D networks used for flow simulations). The thick black and gray curved lines graphically show transforms \#1 and \#2 of Table 3. The values of $z^{*}{ }_{\mathrm{c}}$ corresponding to the percolation threshold (arrow) are indicated. The $z^{*}=z$ line is also shown.

changes in pore size but also by the variations in orientation of the intersected pores with respect to the thin section surface (the chord length histograms reported by Fredrich et al. [1993] are even more sensitive to this orientation effect and therefore cannot be used for our purpose). We obtained lower values, $\sigma_{\text {throat }} \approx 0.6$ and $\sigma_{\text {nodal }} \approx 0.4$, from the threedimensional study of Lindquist et al. [2000] (note also that Baldwin et al. [1996] observed pore radius distributions with normalized standard deviations near 0.3 in beds of uniform spheres). Because $\sigma_{\text {throat }}$ does not integrate the cross-section changes along individual pores while $\sigma_{r} /\langle r\rangle$ does, we argue that $\sigma_{\text {throat }}$ reflects more variability and is therefore larger than $\sigma_{r} /\langle r\rangle$. Accordingly, we assumed that, for Fontainebleau sandstone, $\sigma_{r} /\langle r\rangle$ has a value close to $\sigma_{\text {nodal }}\left(\right.$ i.e., $\sigma_{r} /\langle r\rangle \approx 0.4$ yielding $\beta=2.01$ and $w=0.0366$ ). Presumably, $\sigma_{r} /\langle r\rangle$ of Fontainebleau sandstone may vary with porosity, but we do not have enough data to delineate such a subtle effect. Plugging the above values of $\beta$ and $w$ into equation (4), we calculated theoretical values of $k$ for the completed data sets of Doyen [1988] and Fredrich et al. [1993] and compared them to the experimentally measured values (see Figure 10). We ran the calculations twice, using the estimates of $z$ from (1) Lindquist et al.'s [2000] $z^{*}$ (gray symbols in Figure 10; the error bars represent the difference produced by transforms 1 and 2) and (2) Doyen's [1988] $z_{2 \mathrm{D}}$ (black symbols in Figure 10). As shown in Figure 10, the modeled $k$ values corresponding to $z_{2 \mathrm{D}}$ are in good agreement with the experimental data, whereas those estimated from $z^{*}$ are about one order of magnitude too small. This result is very encouraging, although finding agreement for only one particular rock does not fully establish the validity of the model. We hope additional data on other porous media (including "numerical" materials such as those studied by Sok et al. [2002] and Jones et al. [2009]) will become available in the near future, allowing further testing of our model.

\section{Discussion}

[20] The results of Figure 10 are quite surprising. We expected a comparatively better performance of $z^{*}$, a genuine three-dimensional parameter, than $z_{2 \mathrm{D}}$, an extrapolated quantity. One explanation for the poor performance of $z^{*}$ is that the medial axis method tends to yield values of $z^{*}$ biased toward 3 [Petford et al., 2001]. Indeed, Lindquist et al. [2000] measured $z^{*}$ between 3.37 and 3.75 for samples with $\phi$ from $8.4 \%$ to $21.3 \%$, and Doyen [1988] estimated $z_{2 \mathrm{D}}$ values ranging from 2.3 to 6 for porosities between $5.2 \%$ and $22.1 \%$. Note also that Baldwin et al. [1996] used a different scheme to identify individual pores in three-dimensional images of packed uniform spheres and measured much larger coordination numbers than the values mentioned above (i.e., median $z$ around 6). One exception might be sample F1 from Fredrich et al. [1993] $\left(\phi=4.6 \%, k=0.42 \mathrm{mD}\right.$, or $10-{ }^{15} \mathrm{~m}^{2}$; marked by an arrow in Figures 8 and 10), for which the underprediction is less strong and, in fact, may have been caused by a slightly incorrect choice of the $z^{*}$ versus $z$ transform. Indeed, a minute decrease of $z^{*}$ can produce a significant increase in modeled $k$ in rocks near the percolation threshold. We verified that $z^{*}{ }_{\mathrm{c}}=3.18$, a value barely smaller than the ones selected and nevertheless consistent with Figure 9, did yield a nearly exact prediction of $k$. On the other hand, the reason for the success of Doyen's [1988] 


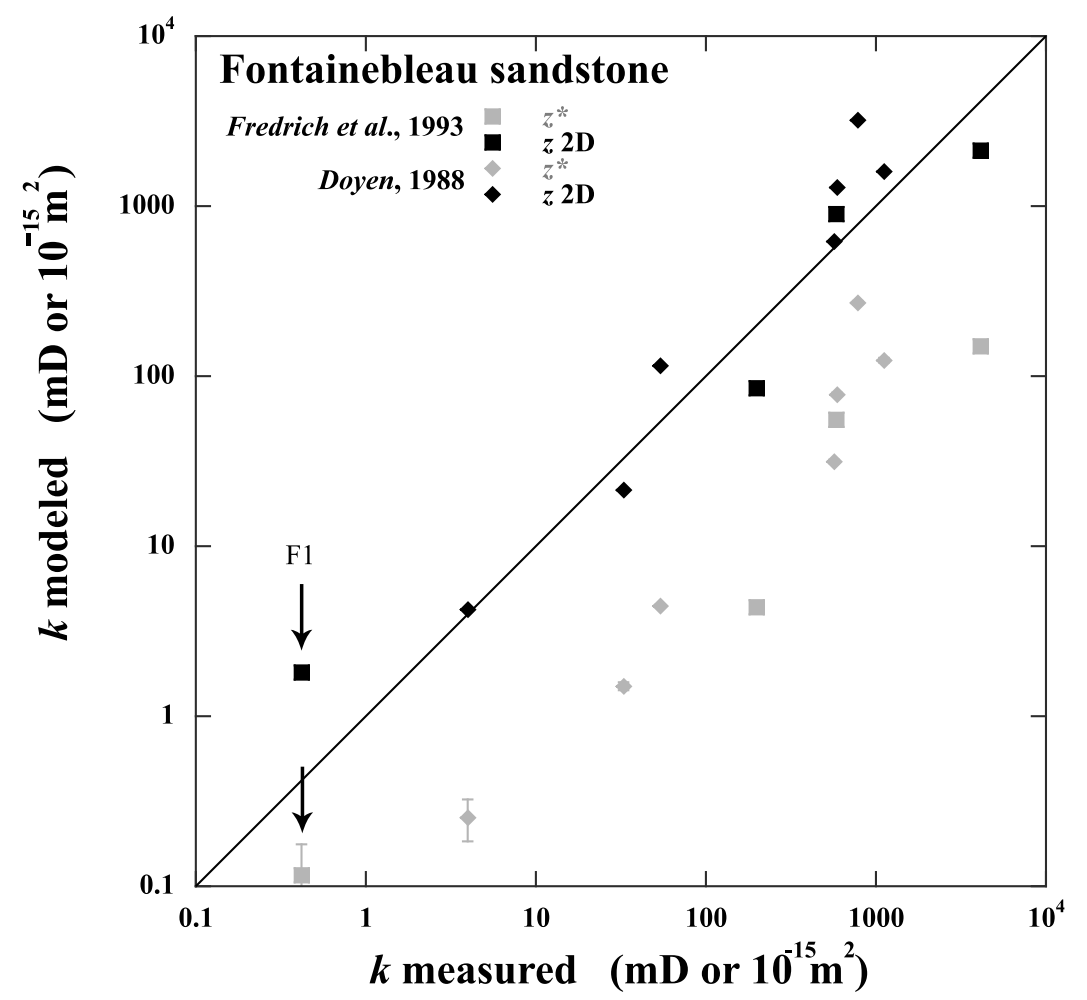

Figure 10. Comparison of the permeabilities modeled using equation (4) to those measured by Doyen [1988] (diamonds) and Fredrich et al. [1993] (squares) in Fontainebleau sandstone samples (see Figure 8). The black symbols correspond to the modeled values on the basis of Doyen's [1988] extrapolated $z_{2 \mathrm{D}}$, whereas the gray ones represent the values obtained using Lindquist et al. [2000] $z^{*}$ (error bars show difference between transform \#1 and transform \#2).

method is hard to assess. He used an erosion algorithm to identify pseudo nodes and branches in two-dimensional micrographs. He then defined a pseudo two-dimensional coordination number as the ratio of total number of branches to total number of nodes in a micrograph. Finally, Doyen [1988] extrapolated the three-dimensional coordination number (here, denoted $z_{2 \mathrm{D}}$ ) by assuming that $z_{2 \mathrm{D}}$ is proportional to the pseudo coordination number and by tying it to a high value, $z_{2 \mathrm{D}}=6$, for the sample with $\phi=22 \%$. This choice was clearly instrumental in generating high coordination numbers. It was justified by claiming that coordination numbers of about 6 occur in close random packing of grains, an assertion confirmed by the study of Baldwin et al. [1996]. Whatever the reason, Doyen's [1988] extrapolation method worked remarkably well. The only exception may again be sample F1 from Fredrich et al. [1993], for which $z_{2 \mathrm{D}}$ was somewhat overestimated. This sample is very close to the percolation threshold and may contain some disconnected porosity (although Song and Renner [2008] measured only negligible unconnected porosity in Fontainebleau sandstones, even for $\phi<6 \%$ ). In our network simulations, this situation corresponds to a substantial number of nodes with a local coordination number of zero (i.e., disconnected nodal pores). The erosion procedure used by Doyen [1988] is likely to generate more pseudo branches than are present in reality and therefore underestimate the number of nodal pores with $z=0$.
[21] Although specific difficulties arise in rocks near a percolation threshold, our results suggest that $z$ is the proper parameter for characterizing pore connectivity in rocks. It is important to recall that the existence of a percolation threshold is not a general feature of rocks (e.g., hot-pressed calcite aggregates become impermeable for a finite value of porosity, whereas this is not true for hot-pressed quartz [Bernabé et al., 2003b]). Thus, introducing a critical porosity $\phi_{\mathrm{c}}$ as is often done is not a general solution for modeling permeability and, in fact, amounts to adding an extra model parameter. On the other hand, $z$ is a true independent variable, and it provides a "universal" model of permeability that remains valid in the entire porosity range whether a percolation threshold exists or not. Note also that no extra parameter is needed to account for a possible percolation threshold; $z_{\mathrm{c}}=1.5$ is simply reached in this case, whereas $z>1.5$ if no percolation threshold exists.

[22] As was pointed out by Fredrich et al. [1993], the strong permeability variations observed in Fontainebleau sandstone cannot be explained solely by the relatively modest changes in hydraulic radius observed. Their conclusion, also reached by Doyen [1988], Fauzi et al. [2002] and others, is that the permeability of Fontainebleau sandstone is primarily controlled by pore connectivity. The good quantitative agreement of our model with the Fontainebleau sandstone data strongly supports this conclusion. One important remark stemming from the rather regular variations of $z_{2 \mathrm{D}}$ with porosity in Fontainebleau sandstone is that 
pore connectivity plays a crucial role not just near the percolation threshold (i.e., $\phi \approx 4 \%$ ) but, in fact, in the entire porosity range. This has important implications for the diagenesis of Fontainebleau sandstone. In addition to porosity reduction processes such as mechanical compression, cementation, and so forth, there must exist a pore closing mechanism active even during the relatively early stages of diagenesis. For example, Zhu et al. [1999] modeled the evolution of permeability of hot-pressed calcite aggregates using a combination of power law creep densification and a tube ovulation mechanism (i.e., unstable pinching off of cylindrical pores with fluctuating cross-sections). The ovulation rate increases with decreasing pore radius, so connectivity loss was observed to be very low during the first stages of hot-pressing and gradually increased to become dominant at porosities of $\sim 10 \%$ and lower. Despite the similarity in permeability evolution of hot-pressed calcite and Fontainebleau sandstone, the high-temperature mechanisms considered by Zhu et al. [1999] may not be relevant to the diagenesis of Fontainebleau sandstone. Nevertheless, we can ascertain that an unstable pore closing mechanism must have been active in Fontainebleau sandstone.

[23] In addition to the diagenesis of Fontainebleau sandstone and the high-temperature compaction of granular aggregates, pore connectivity variations have also been documented in dense rocks subjected to brittle straining (for a review, see Bernabé et al. [2003b]). Pore connectivity changes may also explain the very large pressure sensitivity of permeability of certain tight sandstones [ $\mathrm{Li}$ et al., 2009a, 2009b] or of rough fractures [Cook, 1992]. In this last case, the topological description of the pore space may undergo a transition from medial surface when the fracture is open to medial axis when large number of asperities come into contact and fluid flow is restricted to meandering channels between them [Yang et al., 1995; Pyrak-Nolte and Morris, 2000].

\section{Conclusions}

[24] 1. The power laws $\phi k / k_{0} \propto\left(z-z_{\mathrm{c}}\right)^{\gamma}$ and $k / k_{0} \propto$ $\left(z-z_{\mathrm{c}}\right)^{\beta}$ are obeyed in two- and three-dimensional networks, respectively. These power laws are very nearly "universal" in the sense of percolation theory (i.e., independent of lattice type). The exponents of these power laws as well as the prefactors are strongly dependent on pore size heterogeneity.

[25] 2. A permeability model was inferred from these power laws using the hydraulic radius as length scale and containing three scale-invariant parameters, namely, the mean coordination number, the normalized standard deviation of the pore size distribution and the ratio of pore size to pore separation distance.

[26] 3. The coordination number $z$ appears to be the proper parameter for characterizing pore connectivity in rocks in the entire porosity range, including significantly above a percolation threshold. However, precautions must be taken to ensure that $z$ is appropriately measured in threedimensional images (in particular, strict pore identification rules must be used to avoid a possible bias toward $z=3$ ). We hope that interest in characterizing pore connectivity of rocks will grow in the future and more data will become available.
[27] Acknowledgments. We are grateful to B. Clennell, J. Renner and an anonymous associate editor for their thoughtful reviews of our original manuscript. This work was partially funded by the U.S. Department of Energy under grant DE-FG09-97ER14760 and by the Open Fund PLN0901 of the State Key Laboratory of Oil and Gas Reservoir Geology and Exploitation (Southwest Petroleum University, Chengdu, China). This is IPGP contribution 3009 .

\section{References}

Adler, P. (1992), Porous Media: Geometry and Transports, 544 pp., Butterworth-Heinemann, Boston, Mass.

Baldwin, C. A., A. J. Sederman, M. D. Mantle, P. Alexander, and L. F. Gladden (1996), Determination and characterization of the structure of a pore space from 3D volume images, J. Colloid Interface Sci., 181, 79-92.

Bernabé, Y. (1995), The transport properties of networks of cracks and pores, J. Geophys. Res., 100(B3), 4231-4241.

Bernabé, Y., and C. Bruderer (1998), Effect of the variance of the pore size distribution on the transport properties of heterogeneous networks, J. Geophys. Res., 103(B1), 513-525.

Bernabé, Y., W. F. Brace, and B. Evans (1982), Permeability, porosity and pore geometry of hot-pressed calcite, Mech. Mater., 1, 173-183.

Bernabé, Y., C. Bruderer-Weng, and A. Maineult (2003a), Permeability fluctuations in heterogeneous networks with different dimensionality and topology, J. Geophys. Res., 108(B7), 2351, doi:10.1029/2002JB002326.

Bernabé, Y., U. Mok, and B. Evans (2003b), Permeability-porosity relationships in rock subjected to various evolution processes, Pure Appl. Geophys., 160, 937-960.

Berryman, J. G. (1992a), Effective stress for transport properties of inhomogeneous porous rock, J. Geophys. Res., 97(B12), 17409-17424.

Berryman, J. G. (1992b), Exact effective-stress rules in rock mechanics, Phys. Rev. A, 46, 3307-3311.

Berryman, J. G. (1993), Effective-stress rules for pore-fluid transport in rocks containing two minerals, Int. J. Rock Mech. Min. Sci. Geomech. Abstr., 30, 1165-1168.

Blair, S. C., P. A. Berge, and J. G. Berryman (1996), Using two-point correlation functions to characterize microgeometry and estimate permeabilities of sandstones and porous glass, J. Geophys. Res., 101(B9), 20,359-20,375.

Brace, W. F. (1980), Permeability of crystalline and argillaceous rocks, Int. J. Rock Mech. Min. Sci. Geomech. Abstr., 17, 241-251.

Cook, N. G. W. (1992), Natural joints in rock: mechanical, hydraulic and seismic properties under normal stress, Int. J. Rock Mech. Min. Sci. Geomech. Abstr., 29, 198-223.

David, C., B. Menéndez, and M. Darot (1999), Influence of stress-induced and thermal cracking on physical properties and microstructure of La Peyrate granite, Int. J. Rock Mech. Min. Sci. Geomech. Abstr., 36, 433-448.

Doyen, P. M. (1988), Permeability, conductivity, and pore geometry of sandstone, J. Geophys. Res., 93(B7), 7729-7740.

Dullien, F. A. L. (1979), Porous Media: Fluid Transport and Pore Structure, 396 pp., Academic, New York.

Fauzi, U., A. Hoerdt, and F. M. Neubauer (2002), Influence of coordination number and percolation probability on rock permeability estimation, Geophys. Res. Lett., 29(8), 1237, doi:10.1029/2001GL013414.

Fredrich, J. T. (1999), 3D imaging of porous media using laser scanning confocal microscopy with application to microscale transport processes, Phys. Chem. Earth. Part A Solid Earth Geod., 24, 551-561.

Fredrich, J. T., and W. B. Lindquist (1997), Statistical characterization of the three-dimensional microgeometry of porous media and correlation with macroscopic transport properties, Int. J. Rock Mech. Min. Sci. Geomech. Abstr., 34, paper 085.

Fredrich, J. T., K. H. Greaves, and J. W. Martin (1993), Pore geometry and transport properties of Fontainebleau sandstone, Int. J. Rock Mech. Min. Sci. Geomech. Abstr., 30, 691-697.

Gelhar, L. W. (1993), Stochastic Subsurface Hydrology, 390 pp., PrenticeHall, Englewood Cliffs, N. J.

Guéguen, Y., and J. Dienes (1989), Transport properties of rocks from statistics and percolation, Math. Geol., 21, 1-13.

Johnson, D. L. (1989), Scaling function for dynamic permeability, Phys. Rev. Lett., 63, 580.

Johnson, D. L., and L. M. Schwartz (1989), Unified theory of geometrical effects in transport properties of porous media, Trans. SPWLA Ann. Logging Symp., 30th., paper E, 1-25.

Johnson, D. L., J. Koplik, and R. Dashen (1987), Theory of dynamic permeability and tortuosity in fluid-saturated porous media, J. Fluid Mech., 176, 379-402. 
Jones, A. C., C. H. Arns, D. W. Hutmacher, B. K. Milthorpe, A. P. Sheppard, and M. A. Knackstedt (2009), The correlation of pore morphology, interconnectivity and physical properties of 3D ceramic scaffolds with bone ingrowth, Biomaterials, 30, 1440-1451.

Kirkpatrick, S. (1971), Classical transport in disordered media: scaling and effective medium theories, Phys. Rev. Lett., 27, 1722-1725.

Le Ravalec, M., M. Darot, T. Reuschlé, and Y. Guéguen (1996), Transport properties and microstructural characteristics of a thermally cracked mylonite, Pure Appl. Geophys., 146, 207-227.

Li, M., Y. Bernabé, W.-I. Xiao, Z.-Y. Chen, and Z.-Q. Liu (2009a), Effective pressure law for permeability of E-bei sandstones, J. Geophys. Res., 114, B07205, doi:10.1029/2009JB006373.

Li, M., W. L. Xiao, X. Guo, L. H. Zhang, and L. L. Zheng (2009b), Laboratory study of the effective pressure law for permeability in Ta-Ba-Miao low-permeability sandstones (in Chinese), Chin. J. Geophys., 52(12), 3166-3174, doi:10.3969/j.issn.0001-5733.2009.12.027.

Lindquist, W. B., and A. Venkatarangan (1999), Investigating 3D geometry of porous media from high resolution images, Phys. Chem. Earth, Part A Solid Earth Geod., 24, 593-599.

Lindquist, W. B., S. M. Lee, D. A. Coker, K. W. Jones, and P. Spanne (1996), Medial axis analysis of void structure in three-dimensional tomographic images of porous media, J. Geophys. Res., 101(B4), 8297-8310.

Lindquist, W. B., A. Venkatarangan, J. Dunsmuir, and T-f. Wong (2000), Pore and throat size distributions measured from synchrotron X-ray tomographic images of Fontainebleau sandstones, J. Geophys. Res., 105(B9), 21509-21527.

Madden, T. R. (1983), Microcrack connectivity in rocks: a renormalization group approach to the critical phenomena of conduction and failure in crystalline rocks, J. Geophys. Res., 88(B1), 585-592.

Mavko, G., and A. Nur (1997), The effect of a percolation threshold in the Kozeny-Carman relation, Geophysics, 62, 1480-1482.

Paterson, M. S. (1983), The equivalent channel model for permeability and resistivity in fluid-saturated rocks-a reappraisal, Mech. Mater., 2, 345-352.

Petford, N., G. Davidson, and J. A. Miller (2001), Investigation of the petrophysical properties of a porous sandstone sample using confocal scanning laser microscopy, Pet. Geosci., 7, 99-105.

Pyrak-Nolte, L. J., and J. P. Morris (2000), Single fractures under normal stress: The relation between fracture specific stiffness and fluid flow, Int. J. Rock. Min. Sci., 37, 245-262, doi:10.1016/S1365-1609(99)00104-5.
Revil, A. (1999), Ionic diffusivity, electrical conductivity, membrane and thermoelectric potentials in colloids and granular porous media: a unified model, J. Colloid Interface Sci., 212, 503-522.

Revil, A., and P. W. J. Glover (1997), Theory of ionic surface electrical conduction in porous media, Phys. Rev. B, 55, 1757-1773.

Sahimi, M. (1995), Flow and Transport in Porous Media and Fractured Rock, 482 pp., VCH, New York.

Sok, R. M., M. A. Knackstedt, A. P. Sheppard, W. V. Pinczewski, W. B. Lindquist, and A. Venkatarangan (2002), Direct and stochastic generation of network models from tomographic images: effect of topology on residual saturation, Transp. Porous Media, 46, 345-372.

Song, I., and J. Renner (2008), Hydromechanical properties of Fontainebleau sandstone: experimental determination and micromechanical modeling, J. Geophys. Res., 113, B09211, doi:10.1029/2007JB005055.

Spanne, P., J. F. Thovert, C. J. Jacquin, W. B. Lindquist, K. W. Jones, and P. M. Adler (1994), Synchrotron computed microtomography of porous media: topology and transport, Phys. Rev. Lett., 73, 2001-2004.

Thovert, J. F., F. Yousefian, P. Spanne, C. G. Jacquin, and P. M. Adler (1994), Grain reconstruction of porous media: application to a lowporosity Fontainebleau sandstone, Phys. Rev. E, 63, 061307.

Walsh, J. B., and W. F. Brace (1984), The effect of pressure on porosity and the transport properties of rock, J. Geophys. Res., 89(B11), 9425-9431.

Yang, G., L. R. Myer, S. R. Brown, and N. G. W. Cook (1995), Microscopic analysis of macroscopic transport properties of single natural fractures using graph theory algorithms, Geophys. Res. Lett., 22, 1429-1432.

Zhang, S., M. S. Paterson, and S. F. Cox (1994), Porosity and Permeability evolution during hot isostatic pressing of calcite aggregates, J. Geophys. Res., 99(B8), 15,741-15,775.

Zhu, W., B. Evans, and Y. Bernabé (1999), Densification and permeability reduction in hot-pressed calcite: a kinetic model, J. Geophys. Res., 104(B11), 25501-25511.

Zinszner, B., and F. M. Pellerin (2007), A Geoscientist's Guide to Petrophysics, 384 pp., Technip, Paris, France.

Y. Bernabé, Earth, Atmospheric and Planetary Sciences Department, Massachusetts Institute of Technology, Rm. 54-722, Cambridge, MA 02139, USA. (yvb@mit.edu)

M. Li, State Key Laboratory of Oil and Gas Reservoir Geology and Exploitation, Southwest Petroleum University, Chengdu, 610500 China.

A. Maineult, Institut de Physique du Globe de Paris, CNRS and Université Paris Cité, 1 rue Cuvier, F-75005 Paris, France. 